\title{
Polynomial inverses of 2D transfer matrices and finite memory realizations via inverse systems
}

\author{
Maria Elena Valcher and Ettore Fornasini \\ Dept. of Electronics and Comp.Sci.,University of Padova \\ via Gradenigo 6/A, 35131 PADOVA, Italy
}

\begin{abstract}
Let $G\left(z_{1}, z_{2}\right)$ be a $p \times m 2 \mathrm{D}$ proper rational transfer matrix, with full column rank, and $\Sigma=\left(A_{1}, A_{2}, B_{1}, B_{2}, C, D\right)$ a state space realization of its. Necessary and sufficient conditions are presented in this paper, which guarantee that (i) $G\left(z_{1}, z_{2}\right)$ admits polynomial left inverses, (ii) such polynomial inverses are transfer matrices of some inverse system of $\Sigma$. When the above conditions are not fulfilled, the existence of stable and/or proper, possibly delayed, inverses of $G\left(z_{1}, z_{2}\right)$, is also discussed.
\end{abstract}

\section{Introduction}

The investigation of $2 \mathrm{D}$ inverse systems represents an interesting topic from the point of view of several applications, such as multivariable system decoupling [1] and the synthesis of finite memory decoders [2]. Even more, the inversion of $2 \mathrm{D}$ systems is of great interest from a theoretical point of view. Actually it involves a detailed analysis of the structural properties of polynomial and rational matrices that represent the input-output transfer matrices of $2 \mathrm{D}$ systems, and the study of some connections between $2 \mathrm{D}$ external descriptions and state models.

Given a proper rational transfer matrix $G\left(z_{1}, z_{2}\right), p \times m$ with $p>m$, the full (column) rank condition is necessary and sufficient to guarantee the existence of a rational left inverse, i.e. an $m \times p$ matrix, $G^{-1}\left(z_{1}, z_{2}\right)$, such that

$$
G^{-1}\left(z_{1}, z_{2}\right) G\left(z_{1}, z_{2}\right)=I_{m}
$$

Being a rectangular matrix, $G\left(z_{1}, z_{2}\right)$ admits an infinite number of left inverses, and sometimes this degree of freedom can be used to single out inverse matrices endowed with special properties. 
Perhaps the most common requirement on $G^{-1}\left(z_{1}, z_{2}\right)$ is the properness condition, which corresponds to the quarter plane causality. It's quite clear that the possibility of finding such an inverse is equivalent to the assumption that $G(0,0)$ is full column rank.

Moreover, it can be easily realized that when $G\left(z_{1}, z_{2}\right)$ admits proper left inverses, the existence of a polynomial inverse represents a particularly favourable case: indeed it's associated to a 2D FIR filter and, consequentely, involves a moving average processing of the input data. Such a feature is also of fundamental importance in two-dimensional decoding, in order to avoid the arising of "catastrophic" errors.

An interesting problem arises when $G(0,0)$ is not full column rank, so that is not possible to obtain a proper inverse of $G\left(z_{1}, z_{2}\right)$. Unlike the 1D case, where the introduction of appropriate delay elements always makes possible to properly invert a proper transfer matrix, the $2 \mathrm{D}$ case exhibits some remarkable features. Indeed examples can be given of $2 \mathrm{D}$ transfer matrices for which is not even possible to find a delayed proper inverse, that is a proper rational matrix $H\left(z_{1}, z_{2}\right)$ such that

$$
H\left(z_{1}, z_{2}\right) G\left(z_{1}, z_{2}\right)=z_{1}^{\nu_{1}} z_{2}^{\nu_{2}} I_{m}
$$

See, for instance, $G\left(z_{1}, z_{2}\right)=\left[\begin{array}{ll}z_{1}+z_{2} & z_{1}+z_{2}\end{array}\right]^{T}$.

2D state models considered in this paper have the following structure [3]

$$
\begin{aligned}
x(h+1, k+1) & =A_{1} x(h, k+1)+A_{2} x(h+1, k) \\
& +B_{1} u(h, k+1)+B_{2} u(h+1, k) \\
y(h, k) & =C x(h, k)+D u(h, k)
\end{aligned}
$$

where the local state $x$ is an $n$-dimensional vector over the field $\mathbf{R}$, input and output functions take values in $\mathbf{R}^{m}$ and $\mathbf{R}^{p}$ respectively, and $A_{1}, A_{2}$, $B_{1}, B_{2}, C, D$ are matrices of suitable dimension, with entries in $\mathbf{R}$.

For sake of brevity, model (1.3) will be denoted as $\Sigma=\left(A_{1}, A_{2}, B_{1}, B_{2}, C, D\right)$.

Every $2 \mathrm{D}$ proper rational matrix $G\left(z_{1}, z_{2}\right)$ can be realized by a $2 \mathrm{D}$ state model $[3,4]$, and therefore can be regarded as the input-output map of a model (1.3), for an appropriate choice of matrices $A_{1}, A_{2}, B_{1}, B_{2}, C$ and $D$.

If $D=G(0,0)$ is full rank, we can associate the inverse system, $[5,6]$, $\Sigma^{-1}\left(D^{-1}\right)=\left(A_{1}-B_{1} D^{-1} C, A_{2}-B_{2} D^{-1} C, B_{1} D^{-1}, B_{2} D^{-1},-D^{-1} C, D^{-1}\right)$ to a given realization $\Sigma$ of $G\left(z_{1}, z_{2}\right)$ and to a particular left inverse $D^{-1}$ of $D$. Since $\Sigma^{-1}\left(D^{-1}\right)$ realizes a proper inverse of $G\left(z_{1}, z_{2}\right)$, the problem of finding a causal inverse for a proper transfer matrix can be solved by resorting to an inverse system.

Finite memory 2D systems, $[7,8]$, that is systems (1.3) for which the characteristic polynomial $\operatorname{det}\left(I-A_{1} z_{1}-A_{2} z_{2}\right)$ is a unit, constitute the most "natural" state model for realizing FIR filters, since in this case the FIR 
property is a direct consequence of the zeroing of the free state evolution in a finite number of steps.

When a transfer matrix $G\left(z_{1}, z_{2}\right)$ admits polynomial inverses, we may ask whether inverse possibly finite memory, state models allow to realize them.

Actually, even though the inversion of a state model always produces a proper inverse of $G\left(z_{1}, z_{2}\right)$, nevertheless not every proper inverse of $G\left(z_{1}, z_{2}\right)$ can be obtained in this way. In particular, examples can be given given of matrices which have polynomial inverses but none of them can be realized by an inverse system.

The aim of this paper is to provide a fairly detailed discussion of the problems mentioned above, and to present some new results. In sections 2 and 3, necessary and sufficient conditions are given for a proper rational matrix to have polynomial inverses and, in particular, polynomial inverses realizable through an inverse $2 \mathrm{D}$ system. Section 4 deals with the singular case, (i.e. $G(0,0)$ not full rank). The existence of a delayed proper inverse is shown to be equivalent to a condition on the variety $\mathcal{V}(G)$ of the maximal order minors of $G\left(z_{1}, z_{2}\right)$. The special case of a polynomial delayed inverse is also considered, and reduced to a further condition on $\mathcal{V}(G)$, that must be a subset of the coordinate axes of $\mathbf{C} \times \mathbf{C}$.

\section{Polynomial inverses of $2 \mathrm{D}$ transfer matrices}

Throughout this paper, we will assume that $G\left(z_{1}, z_{2}\right)$ is a $2 \mathrm{D}$ proper rational transfer matrix, $p \times m$ with $p>m$ and rank $m$ over the field of rational functions $\mathbf{R}\left(z_{1}, z_{2}\right)$. Moreover in sections 2 and 3 we will also assume $G(0,0)$ full column rank, which implies that the set

$$
\begin{gathered}
\mathcal{S}(G):=\left\{\Sigma=\left(A_{1}, A_{2}, B_{1}, B_{2}, C, D\right): C\left(I-A_{1} z_{1}-A_{2} z_{2}\right)^{-1}\right. \\
\left.\left(B_{1} z_{1}+B_{2} z_{2}\right)+D=G\left(z_{1}, z_{2}\right)\right\}
\end{gathered}
$$

of all the state models which realize the transfer matrix $G\left(z_{1}, z_{2}\right)$ consists of invertible systems. Given $\Sigma=\left(A_{1}, A_{2}, B_{1}, B_{2}, C, D\right)$ in $\mathcal{S}(G)$ and a left inverse, $D^{-1}$, of $D$, the inverse system $\Sigma^{-1}\left(D^{-1}\right)=\left(A_{1}-B_{1} D^{-1} C, A_{2}-\right.$ $\left.B_{2} D^{-1} C, B_{1} D^{-1}, B_{2} D^{-1},-D^{-1} C, D^{-1}\right)$ realizes a proper left inverse of $G\left(z_{1}, z_{2}\right)$. In general, however, the inverse systems belonging to

$$
\mathcal{S}^{-1}(G):=\left\{\Sigma^{-1}\left(D^{-1}\right): \Sigma \in \mathcal{S}(G), D^{-1} D=I_{m}\right\}
$$

do not realize all the left inverses of $G\left(z_{1}, z_{2}\right)$.

In this section we derive necessary and sufficient conditions, guaranteeing that a proper rational matrix (in particular a polynomial one), $G\left(z_{1}, z_{2}\right)$, 
admits a polynomial inverse $G^{-1}\left(z_{1}, z_{2}\right)$. We will determine in which cases $\mathcal{S}^{-1}(G)$ includes systems having a polynomial transfer matrix, and in particular finite memory systems.

Let $N_{R} D_{R}^{-1}$ be a right coprime matrix fraction description (rcMFD) of $G\left(z_{1}, z_{2}\right),[9,10]$, and suppose, without loss of generality, that $D_{R}(0,0)=I_{m}$, so that

$$
G(0,0)=N_{R}(0,0) D_{R}^{-1}(0,0)=N_{R}(0,0)
$$

Under these assumptions we can prove the following proposition.

Proposition 1. a) $G\left(z_{1}, z_{2}\right)$ admits a polynomial inverse if and only if there is a polynomial matrix $Q\left(z_{1}, z_{2}\right)$ such that

$$
D_{R}\left(z_{1}, z_{2}\right)=Q\left(z_{1}, z_{2}\right) N_{R}\left(z_{1}, z_{2}\right)
$$

b) If $\Sigma=\left(A_{1}, A_{2}, B_{1}, B_{2}, C, D\right)$ realizes the transfer matrix $G\left(z_{1}, z_{2}\right)$, the inverse system $\Sigma^{-1}\left(D^{-1}\right)$ realizes a polynomial inverse of $G\left(z_{1}, z_{2}\right)$ if and only if there is a polynomial matrix $P\left(z_{1}, z_{2}\right)$ such that

$$
D_{R}\left(z_{1}, z_{2}\right)=P\left(z_{1}, z_{2}\right) D^{-1} N_{R}\left(z_{1}, z_{2}\right)
$$

c) $\Sigma^{-1}\left(D^{-1}\right)$ is finite memory if and only if $D^{-1} N_{R}\left(z_{1}, z_{2}\right)$ is a unimodular matrix and $\Sigma$ is a coprime realization of $G\left(z_{1}, z_{2}\right)$.

\section{PROOF}

a) If $Q\left(z_{1}, z_{2}\right)$ is a polynomial left inverse of $G\left(z_{1}, z_{2}\right)$, postmultiplying both the members of

$$
Q\left(z_{1}, z_{2}\right) N_{R}\left(z_{1}, z_{2}\right) D_{R}^{-1}\left(z_{1}, z_{2}\right)=I_{m}
$$

by $D_{R}\left(z_{1}, z_{2}\right)$, we obtain $(2.3)$.

Conversely, if we postmultiply both the sides of (2.3) by $D_{R}^{-1}\left(z_{1}, z_{2}\right)$, we obtain

$$
Q\left(z_{1}, z_{2}\right) N_{R}\left(z_{1}, z_{2}\right) D_{R}^{-1}\left(z_{1}, z_{2}\right)=I_{m} .
$$

So $Q\left(z_{1}, z_{2}\right)$ is a polynomial inverse of $G\left(z_{1}, z_{2}\right)$.

b) Rewriting the transfer matrix of $\Sigma^{-1}\left(D^{-1}\right)$ as:

$$
\begin{aligned}
& G^{-1}\left(z_{1}, z_{2}\right)=-D^{-1} C\left[I-A_{1} z_{1}-A_{2} z_{2}+\left(B_{1} z_{1}+B_{2} z_{2}\right) D^{-1} C\right]^{-1} \\
& \left(B_{1} z_{1}+B_{2} z_{2}\right) D^{-1}+D^{-1} \\
& =-D^{-1} C\left(I+\left(I-A_{1} z_{1}-A_{2} z_{2}\right)^{-1}\left(B_{1} z_{1}+B_{2} z_{2}\right) D^{-1} C\right)^{-1} \\
& \left(I-A_{1} z_{1}-A_{2} z_{2}\right)^{-1}\left(B_{1} z_{1}+B_{2} z_{2}\right) D^{-1}+D^{-1}
\end{aligned}
$$

and using the identity $(I+H S)^{-1} H=H(I+S H)^{-1}$, we obtain

$G^{-1}\left(z_{1}, z_{2}\right)=-D^{-1} C\left(I-A_{1} z_{1}-A_{2} z_{2}\right)^{-1}\left(B_{1} z_{1}+B_{2} z_{2}\right) D^{-1}[I+C(I-$ $\left.\left.A_{1} z_{1}-A_{2} z_{2}\right)^{-1}\left(B_{1} z_{1}+B_{2} z_{2}\right) D^{-1}\right]^{-1}+D^{-1}$ 


$$
\begin{aligned}
& =-D^{-1}\left[G\left(z_{1}, z_{2}\right)-D\right]\left[D^{-1} G\left(z_{1}, z_{2}\right)\right]^{-1} D^{-1}+D^{-1} \\
& =\left[\left(-D^{-1} G\left(z_{1}, z_{2}\right)+I\right)\left(D^{-1} G\left(z_{1}, z_{2}\right)\right)^{-1}+I\right] D^{-1} \\
& =\left[D^{-1} G\left(z_{1}, z_{2}\right)\right]^{-1} D^{-1}
\end{aligned}
$$

It follows that $G^{-1}\left(z_{1}, z_{2}\right)$ is polynomial if and only if

$$
\left[D^{-1} G\left(z_{1}, z_{2}\right)\right]^{-1} D^{-1}=\left[D^{-1} N_{R}\left(z_{1}, z_{2}\right) D_{R}^{-1}\left(z_{1}, z_{2}\right)\right]^{-1} D^{-1},
$$

has the same property. Postmultiplying (2.5) by $D$, we can see that (2.5) is polynomial if and only if $\left[D^{-1} N_{R}\left(z_{1}, z_{2}\right) D_{R}^{-1}\left(z_{1}, z_{2}\right)\right]^{-1}$, and hence

$$
P\left(z_{1}, z_{2}\right):=D_{R}\left(z_{1}, z_{2}\right)\left(D^{-1} N_{R}\left(z_{1}, z_{2}\right)\right)^{-1}
$$

are polynomial.

From (2.6) we obtain (2.4) and viceversa.

c) The system $\Sigma^{-1}\left(D^{-1}\right)$ is finite memory if and only if

$$
\begin{aligned}
1 & =\operatorname{det}\left[I-\left(A_{1}-B_{1} D^{-1} C\right) z_{1}-\left(A_{2}-B_{2} D^{-1} C\right) z_{2}\right] \\
& =\operatorname{det}\left(I-A_{1} z_{1}-A_{2} z_{2}\right) \operatorname{det}\left[I+\left(I-A_{1} z_{1}-A_{2} z_{2}\right)^{-1}\left(B_{1} z_{1}+B_{2} z_{2}\right) D^{-1} C\right] \\
& =\operatorname{det}\left(I-A_{1} z_{1}-A_{2} z_{2}\right) \operatorname{det}\left[I+D^{-1} C\left(I-A_{1} z_{1}-A_{2} z_{2}\right)^{-1}\left(B_{1} z_{1}+B_{2} z_{2}\right)\right] \\
& =\operatorname{det}\left(I-A_{1} z_{1}-A_{2} z_{2}\right) \operatorname{det}\left[D^{-1} N_{R}\left(z_{1}, z_{2}\right) D_{R}^{-1}\left(z_{1}, z_{2}\right)\right] \\
& =\operatorname{det}\left(I-A_{1} z_{1}-A_{2} z_{2}\right) \operatorname{det}\left[D^{-1} N_{R}\left(z_{1}, z_{2}\right)\right] / \operatorname{det} D_{R}\left(z_{1}, z_{2}\right)
\end{aligned}
$$

that is if and only if

$$
\operatorname{det} D_{R}\left(z_{1}, z_{2}\right)=\operatorname{det}\left(I-A_{1} z_{1}-A_{2} z_{2}\right) \operatorname{det}\left[D^{-1} N_{R}\left(z_{1}, z_{2}\right)\right]
$$

Keeping in mind that $\operatorname{det} D_{R}\left(z_{1}, z_{2}\right)$ is a divisor of $\operatorname{det}\left(I-A_{1} z_{1}-A_{2} z_{2}\right)$, and the two polynomials coincide if and only if $\Sigma$ is a coprime realization, it follows that (2.7) holds if and only if

1) $\Sigma$ is a coprime realization of $G\left(z_{1}, z_{2}\right)$;

2) $\operatorname{det}\left[D^{-1} N_{R}\left(z_{1}, z_{2}\right)\right]=1$, so $D^{-1} N_{R}\left(z_{1}, z_{2}\right)$ is a unimodular matrix.

Remark I : If $N_{R}\left(z_{1}, z_{2}\right)$ is zero-prime, (2.3) is fulfilled by assuming $Q\left(z_{1}, z_{2}\right)=\left[D_{R}\left(z_{1}, z_{2}\right) N_{R}^{-1}\left(z_{1}, z_{2}\right)\right]$, where $N_{R}^{-1}\left(z_{1}, z_{2}\right)$ is any polynomial left inverse of $N_{R}\left(z_{1}, z_{2}\right)$.

It's worthwhile to underline that a realization $\Sigma\left(D^{-1}\right)$ is finite memory if and only if the following two conditions are simultaneously satisfied:

i) the realization $\Sigma$ is coprime. This property is a characteristic of the state model, and is always obtainable by resorting to an appropriate choice of the realization of $G\left(z_{1}, z_{2}\right)$;

ii) $D^{-1} N_{R}\left(z_{1}, z_{2}\right)=G(0,0)^{-1} N_{R}\left(z_{1}, z_{2}\right)$ is unimodular. This property depends only on the transfer matrix $G\left(z_{1}, z_{2}\right)$ and the choice of $D^{-1}$. 
The unimodularity of $D^{-1} N_{R}\left(z_{1}, z_{2}\right)$ constitutes a sufficient (but not necessary) condition for $\Sigma^{-1}\left(D^{-1}\right)$ to realize a polynomial inverse, since (2.4) holds with $P\left(z_{1}, z_{2}\right)=D_{R}\left(z_{1}, z_{2}\right)\left[D^{-1} N_{R}\left(z_{1}, z_{2}\right)\right]^{-1}$.

Let's now restrict to the case of $G\left(z_{1}, z_{2}\right)$ being a polynomial matrix. Thus, choosing

$$
\begin{aligned}
& N_{R}\left(z_{1}, z_{2}\right)=G\left(z_{1}, z_{2}\right) \\
& D_{R}\left(z_{1}, z_{2}\right)=I_{m}
\end{aligned}
$$

the previous proposition can be restated as follows.

Proposition 2. Let $\Sigma=\left(A_{1}, A_{2}, B_{1}, B_{2}, C, D\right)$ be a realization of a $2 \mathrm{D}$ polynomial transfer matrix $G\left(z_{1}, z_{2}\right)$ and $D^{-1}$ a left inverse of $D$. The inverse system $\Sigma^{-1}\left(D^{-1}\right)$ realizes a polynomial inverse of $G\left(z_{1}, z_{2}\right)$, if and only if $D^{-1} G\left(z_{1}, z_{2}\right)$ is unimodular. If that condition is met, $\Sigma^{-1}\left(D^{-1}\right)$ is a finite memory system if and only if $\Sigma$ has the same property.

\section{PROOF}

From the proof of Proposition 1, we know that the transfer matrix of $\Sigma^{-1}\left(D^{-1}\right)$ is

$$
G^{-1}\left(z_{1}, z_{2}\right)=\left[D^{-1} G\left(z_{1}, z_{2}\right)\right]^{-1} D^{-1} .
$$

Obviously $\left[D^{-1} G\left(z_{1}, z_{2}\right)\right]^{-1}$ is polynomial if and only if $\left[D^{-1} G\left(z_{1}, z_{2}\right)\right]^{-1}$ is polynomial too, and then $D^{-1} G\left(z_{1}, z_{2}\right)$ is a unimodular matrix.

Moreover, keeping in mind (2.8), the finite memory condition (2.7) on $\Sigma^{-1}\left(D^{-1}\right)$ is equivalent to

$$
1=\operatorname{det}\left(I-A_{1} z_{1}-A_{2} z_{2}\right) \operatorname{det}\left[D^{-1} G\left(z_{1}, z_{2}\right)\right]
$$

(2.9) holds if and only if

1) $\operatorname{det}\left[D^{-1} G\left(z_{1}, z_{2}\right)\right]=1$, that is $\Sigma^{-1}\left(D^{-1}\right)$ realizes a polynomial inverse of $G\left(z_{1}, z_{2}\right)$;

2) $\operatorname{det}\left(I-A_{1} z_{1}-A_{2} z_{2}\right)=1$, that is $\Sigma$ is a finite memory realization of $G\left(z_{1}, z_{2}\right)$

Remark II : Proposition 2 implies that if $\Sigma^{-1}\left(D^{-1}\right)$ realizes a polynomial inverse then the polynomial matrix $G\left(z_{1}, z_{2}\right)$ is zero-prime. Indeed it's a well-known result $([10],[11])$ that polynomial matrices with polynomial inverse are zero-prime.

The zero-primeness of $G\left(z_{1}, z_{2}\right)$ is a necessary condition for $D^{-1} G\left(z_{1}, z_{2}\right)$ to be a unimodular matrix: if the maximal order minors of $G\left(z_{1}, z_{2}\right)$ had a common zero, $\left(\alpha_{1}, \alpha_{2}\right) \in \mathbf{C} \times \mathbf{C}$, then $G\left(\alpha_{1}, \alpha_{2}\right)$, and consequentely $D^{-1} G\left(\alpha_{1}, \alpha_{2}\right)$, would have rank less than $m$, and $\operatorname{det}\left[D^{-1} G\left(\alpha_{1}, \alpha_{2}\right)\right]$ would be zero, a contradiction. As it's clearly shown in Example 1, this is not a 
sufficient condition. Therefore, even if $G\left(z_{1}, z_{2}\right)$ is zero-prime, is not generally true that a polynomial inverse of its can be represented as the inverse system transfer matrix of a realization of $G\left(z_{1}, z_{2}\right)$.

A further consequence, [12], of Proposition 2 is that the possibility of obtaining an inverse of $G\left(z_{1}, z_{2}\right)$ via an inverse system $\Sigma^{-1}\left(D^{-1}\right)$ is independent of the specific realization $\Sigma$, and relies only on $G\left(z_{1}, z_{2}\right)$ and the particular inverse matrix choosen for $D$. Only when we introduce the additional requirement of finite memory, the internal properties of the realization $\Sigma$ become relevant.

Example 2 shows how different choices of $D^{-1}$ lead to different results as regards the unimodularity of $D^{-1} G\left(z_{1}, z_{2}\right)$.

\section{Example 1 :}

Consider the $4 \times 1$ zero-prime matrix

$$
G\left(z_{1}, z_{2}\right)=\left[\begin{array}{c}
1+z_{1} \\
z_{1} z_{2} \\
1+z_{2} \\
z_{1}^{2}
\end{array}\right]
$$

with $D=G(0,0)=[1,0,1,0]^{T}$.

All left inverses of $D$ can be represented as

$$
D^{-1}=[a+1, b,-a, c] \quad a, b, c \in \mathbf{R}
$$

but for every choice of the real parameters $a, b$, and $c$

$$
D^{-1} G\left(z_{1}, z_{2}\right)=1+(a+1) z_{1}+b z_{1} z_{2}-a z_{2}+c z_{1}^{2}
$$

is not unitary.

\section{Example 2 :}

Consider the $4 \times 1$ zero-prime matrix

$$
G\left(z_{1}, z_{2}\right)=\left[\begin{array}{c}
z_{1}+1 \\
z_{1}+2 \\
z_{2} \\
0
\end{array}\right]
$$

with $D=G(0,0)=[1,2,0,0]^{T}$. If we choose the row vector

$$
D^{-1}=[1,0,0,0]
$$


as a left inverse for $D$, the condition $\operatorname{det}\left[D^{-1} G\left(z_{1}, z_{2}\right)\right]=1$ is not satisfied, while it is for

$$
D^{-1}=[-1,1,0,3] \text {. }
$$

\section{Inversion of zero-prime polynomial transfer ma- trices via inverse systems}

As proved in Proposition 2, given a realization $\Sigma$ of a zero-prime polynomial matrix $G\left(z_{1}, z_{2}\right)$, it's possible to obtain a polynomial left inverse of $G\left(z_{1}, z_{2}\right)$ through the inverse system $\Sigma^{-1}\left(D^{-1}\right)$ if and only if $D^{-1} G\left(z_{1}, z_{2}\right)$ is a unimodular matrix. In this section we will study some structural conditions on a zero-prime polynomial matrix $G\left(z_{1}, z_{2}\right)$ which ensure the unimodularity of $D^{-1} G\left(z_{1}, z_{2}\right)$.

Particularly interesting from an algebraic point of view, is the possibility of column bordering up $G\left(z_{1}, z_{2}\right)$ into a unimodular $p \times p$ matrix by an appropriate set of $p-m$ constant columns, as stated in Proposition 3. Since the Quillen-Suslin Theorem [9] guarantees that every $p \times m$ zeroprime polynomial matrix $G\left(z_{1}, z_{2}\right)$ can be included as the first $\mathrm{m}$ columns of some $p \times p$ unimodular matrix, the polynomial transfer matrices for which a polynomial inverse can be obtained through an inverse system, can be equivalentely characterized as those which have a constant Quillen-Suslin completion.

Proposition 3 . Let $G\left(z_{1}, z_{2}\right)$ be a zero-prime polynomial matrix, $p \times m$ with $p>m$, and $D^{-1}$ a left inverse of $G(0,0)=D$. The following statements are equivalent:

i) $D^{-1} G\left(z_{1}, z_{2}\right)$ is a unimodular matrix;

ii) $G\left(z_{1}, z_{2}\right)$ can be expressed as follows:

$$
G\left(z_{1}, z_{2}\right)=D U\left(z_{1}, z_{2}\right)+B\left(z_{1}, z_{2}\right)
$$

where $U\left(z_{1}, z_{2}\right)$ is a unimodular matrix with $U(0,0)=I_{m}, B\left(z_{1}, z_{2}\right)$ a polynomial matrix with $B(0,0)=0$ and

$$
D^{-1} B\left(z_{1}, z_{2}\right)=0
$$

iii) $G\left(z_{1}, z_{2}\right)$ can be column-bordered up into a unimodular matrix, $M\left(z_{1}, z_{2}\right)$, by resorting to a $p \times(p-m)$ constant matrix $X$ :

$$
M\left(z_{1}, z_{2}\right)=\left[G\left(z_{1}, z_{2}\right) X\right]
$$

such that $[D X]$ is invertible and $D^{-1} X=0$. 
PROOF

$i) \Rightarrow i i)$ : Let $A$ a $(p-m) \times p$ constant matrix which completes $D^{-1}$ to a full rank square matrix and is orthogonal to $D$; then we have

$$
\left[\begin{array}{c}
D^{-1} \\
A
\end{array}\right] G\left(z_{1}, z_{2}\right)=\left[\begin{array}{l}
U\left(z_{1}, z_{2}\right) \\
T\left(z_{1}, z_{2}\right)
\end{array}\right]
$$

where $T\left(z_{1}, z_{2}\right)$ is a polynomial matrix which is zero in $(0,0)$, and $U\left(z_{1}, z_{2}\right)=$ $D^{-1} G\left(z_{1}, z_{2}\right)$ is unimodular with $U(0,0)=I_{m}$.

Let $\left[\begin{array}{ll}D & X\end{array}\right]$ be the inverse of $\left[\begin{array}{c}D^{-1} \\ A\end{array}\right]$ : it follows that

$$
G\left(z_{1}, z_{2}\right)=D U\left(z_{1}, z_{2}\right)+X T\left(z_{1}, z_{2}\right)
$$

and assuming $B\left(z_{1}, z_{2}\right)=X T\left(z_{1}, z_{2}\right)$, we obtain $B(0,0)=0$ and $D^{-1} B\left(z_{1}, z_{2}\right)=$ $D^{-1} X T\left(z_{1}, z_{2}\right)=0$.

ii $) \Rightarrow$ iii $)$ : Suppose that $(3.1)$ holds and $X$ is a $p \times(p-m)$ constant matrix such that $[D X]$ is a full rank square matrix and $D^{-1} X=0$.

Letting $\left[\begin{array}{c}D^{-1} \\ A\end{array}\right]=\left[\begin{array}{ll}D & X\end{array}\right]^{-1}$, we have:

$$
\left[\begin{array}{c}
D^{-1} \\
A
\end{array}\right]\left[G\left(z_{1}, z_{2}\right) X\right]=\left[\begin{array}{cc}
D^{-1} G\left(z_{1}, z_{2}\right) & 0 \\
A G\left(z_{1}, z_{2}\right) & I
\end{array}\right]=\left[\begin{array}{cc}
U\left(z_{1}, z_{2}\right) & 0 \\
A G\left(z_{1}, z_{2}\right) & I
\end{array}\right]
$$

so

$$
\left[G\left(z_{1}, z_{2}\right) X\right]=[D X]\left[\begin{array}{cc}
U\left(z_{1}, z_{2}\right) & 0 \\
A G\left(z_{1}, z_{2}\right) & I
\end{array}\right] .
$$

Thus $\left[G\left(z_{1}, z_{2}\right) X\right]$ is a unimodular matrix, since it's the product of two unimodular matrices.

iii $) \Rightarrow i)$ : Assume that $\left[G\left(z_{1}, z_{2}\right) X\right]$ is unimodular and let $\left[\begin{array}{c}D^{-1} \\ A\end{array}\right]=$ $[D X]^{-1}=[G(0,0) X]^{-1}$. So in

$$
\left[\begin{array}{c}
D^{-1} \\
A
\end{array}\right]\left[G\left(z_{1}, z_{2}\right) X\right]=\left[\begin{array}{cc}
D^{-1} G\left(z_{1}, z_{2}\right) & 0 \\
A G\left(z_{1}, z_{2}\right) & I
\end{array}\right]
$$

the matrix on the right-hand side, and hence $D^{-1} G\left(z_{1}, z_{2}\right)$, are unimodular

\section{Example 3 :}

Let's consider the $4 \times 2$ zero-prime transfer matrix

$$
G\left(z_{1}, z_{2}\right)=\left[\begin{array}{cc}
1+z_{1} & z_{2}^{2} \\
z_{1} & 1+z_{2} \\
-z_{1} & -z_{2}^{2} \\
z_{1}^{2} & -z_{2}
\end{array}\right]
$$


with

$$
G(0,0)=D=\left[\begin{array}{ll}
1 & 0 \\
0 & 1 \\
0 & 0 \\
0 & 0
\end{array}\right]
$$

i) If we assume $D^{-1}=\left[\begin{array}{ll}I_{2} & I_{2}\end{array}\right]$ as left inverse of $D$, we meet the condition $\operatorname{det}\left[D^{-1} G\left(z_{1}, z_{2}\right)\right]=1$.

ii) $G\left(z_{1}, z_{2}\right)$ can be expressed as in (3.1), choosing

$$
U\left(z_{1}, z_{2}\right)=\left[\begin{array}{cc}
1 & 0 \\
z_{1}+z_{1}^{2} & 1
\end{array}\right] \quad B\left(z_{1}, z_{2}\right)=\left[\begin{array}{cc}
z_{1} & z_{2}^{2} \\
-z_{1}^{2} & z_{2} \\
-z_{1} & -z_{2}^{2} \\
z_{1}^{2} & -z_{2}
\end{array}\right]
$$

iii) Following the procedure given in the proof of Proposition 3, select a constant matrix $X$ such that $D^{-1} X=0$ and $[D X]$ is a full rank square matrix. Both conditions are met by the matrix

$$
X=\left[\begin{array}{cc}
-1 & 0 \\
0 & -1 \\
1 & 0 \\
0 & 1
\end{array}\right]
$$

and it's easy to verify that $\left[G\left(z_{1}, z_{2}\right) X\right]$ is unimodular.

If $G\left(z_{1}, z_{2}\right)$ is a column matrix, conditions $\left.\left.i\right) \div i i i\right)$ of the previous proposition can be restated as conditions of linear independence of real valued vectors.

Indeed, given a $p \times 1$ polynomial matrix $G\left(z_{1}, z_{2}\right)$ the unimodularity of $D^{-1} G\left(z_{1}, z_{2}\right)$ reduces to $D^{-1} G\left(z_{1}, z_{2}\right)=1$. So we have to find under which conditions $G\left(z_{1}, z_{2}\right)$ has a left inverse that is a left inverse of $D$, too.

Decomposing $G\left(z_{1}, z_{2}\right)$ into the sum of a constant and a strictly proper part

$$
G\left(z_{1}, z_{2}\right)=G_{s p}\left(z_{1}, z_{2}\right)+G(0,0)=G_{s p}\left(z_{1}, z_{2}\right)+D
$$

we can see that $\operatorname{det}\left[D^{-1} G\left(z_{1}, z_{2}\right)\right]=1$ is satisfied if and only if there's a left inverse of $D$ such that

$$
D^{-1} G_{s p}\left(z_{1}, z_{2}\right)=0
$$

Developing $G_{s p}\left(z_{1}, z_{2}\right)$ as a vector coefficients polynomial:

$$
G_{s p}\left(z_{1}, z_{2}\right)=G_{10} z_{1}+G_{01} z_{2}+G_{11} z_{1} z_{2}+\ldots+G_{d q} z_{1}^{d} z_{2}^{q}
$$

we obtain the following conditions:

$$
D^{-1} G_{i j}=0 \quad 0 \leq i \leq d, \quad 0 \leq j \leq q, \quad i+j>0
$$


We distinguish two cases:

a) $D$ belongs to $S\left(G_{s p}\right):=\operatorname{span}\left\{G_{i j}, 0 \leq i \leq d, 0 \leq j \leq q, i+j>0\right\}$. If $D^{-1}$ satisfies (3.4), it's orthogonal to $S\left(G_{s p}\right)$, and hence to $D$, which contradicts $D^{-1} D=1$;

b) $D$ does not belong to $S\left(G_{s p}\right)$. Then there exists a vector $\mathbf{v}$ in $\left(S\left(G_{s p}\right)\right)^{\perp}$, such that $\mathbf{v}^{T} D=c \neq 0$. Therefore the vector $\mathbf{w}=\mathbf{v} / c$ satisfies the conditions $\mathbf{w}^{T} D=1$ and $\mathbf{w}^{T} G_{i j}=0$ and we can choose $\mathbf{w}^{T}$ as a left inverse of $D$.

The previous remarks are summarized in the following:

Proposition 4 . For a $p \times 1$ polynomial transfer matrix $G\left(z_{1}, z_{2}\right)$ the condition

$$
D^{-1} G\left(z_{1}, z_{2}\right)=1
$$

is satisfied if and only if $D$ does not belong to $S\left(G_{s p}\right)$.

\section{Example 4}

With reference to the column matrix $G\left(z_{1}, z_{2}\right)$ of Example 2:

$D=\left[\begin{array}{llll}1 & 2 & 0 & 0\end{array}\right], G_{10}=\left[\begin{array}{llll}1 & 1 & 0 & 0\end{array}\right]^{T}, G_{01}=\left[\begin{array}{llll}0 & 0 & 1 & 0\end{array}\right]^{T}$, so $D$ does not belong to $S\left(G_{s p}\right)$ and the equation $D^{-1} G\left(z_{1}, z_{2}\right)=1$, in the unknown $D^{-1}$, admits $D^{-1}=\left[\begin{array}{llll}-1 & 1 & 0 & 3\end{array}\right] \in$ to $\left(\operatorname{span}\left(G_{10}, G_{01}\right)\right)^{\perp}$, as a solution.

Remark III : Zero-primeness of $G\left(z_{1}, z_{2}\right)$ is a necessary condition for the unimodularity of $D^{-1} G\left(z_{1}, z_{2}\right)$ and hence for $D$ not to belong to $S\left(G_{s p}\right)$. Indeed if the latter condition is met, the system of equations

$$
G_{10} y_{10}+G_{01} y_{01}+G_{11} y_{11}+\cdots+G_{d q} y_{d q}=-D
$$

is devoid of solutions; then, in particular, the non linear system

$$
G_{10} z_{1}+G_{01} z_{2}+G_{11} z_{1} z_{2}+\cdots+G_{d q} z_{1}^{d} z_{2}^{q}=-D
$$

has no solution. So $G\left(z_{1}, z_{2}\right)$ is different from zero for every $\left(z_{1}, z_{2}\right) \in \mathbf{C} \times \mathbf{C}$.

The results of Proposition 4 partially extend to the case $m>1$ if we consider the maximal order minors instead of the elements of matrix $G\left(z_{1}, z_{2}\right)$. Indeed, by the Cauchy-Binet Theorem, condition $\operatorname{det}\left[D^{-1} G\left(z_{1}, z_{2}\right)\right]=1$ can be expressed as:

$$
1=\sum_{i=1}^{\left(\begin{array}{c}
p \\
m
\end{array}\right)} m_{i}\left(D^{-1}\right) g_{i}\left(z_{1}, z_{2}\right)
$$


where the sum is extended to all the products of the maximal order minors, $m_{i}\left(D^{-1}\right)$, of $D^{-1}$ into the corresponding minors $g_{i}\left(z_{1}, z_{2}\right)$ of $G\left(z_{1}, z_{2}\right)$.

It is then immediate to verify the following:

Proposition 5 . Let

$$
g\left(z_{1}, z_{2}\right)=\left[g_{1}\left(z_{1}, z_{2}\right) \ldots g_{N}\left(z_{1}, z_{2}\right)\right]^{T}
$$

be the column vector of the maximal order minors of $G\left(z_{1}, z_{2}\right)$ and $g_{i j} \in$ $\mathbf{R}^{N}, N=\left(\begin{array}{c}p \\ m\end{array}\right)$, the coefficients of the monomials $z_{1}^{i} z_{2}^{j}$ in $g\left(z_{1}, z_{2}\right)$. A necessary condition for the existence of a left inverse $D^{-1}$ of $D$ such that $\operatorname{det}\left[D^{-1} G\left(z_{1}, z_{2}\right)\right]=1$, is that the vector of the maximal order minors of $D$

$$
g_{00}=m(D)=\left[m_{1}(D) \ldots m_{N}(D)\right]^{T} \in \mathbf{R}^{N}
$$

does not belong to the space $\operatorname{span}\left\{g_{i j}, i+j>0\right\}$.

Condition (3.5), considered as a Bézout identity, guarantees that $G\left(z_{1}, z_{2}\right)$ is zero-prime; moreover it represents a particular example of that identity, since it can be satisfied using constant coefficients $m_{i}\left(D^{-1}\right)$.

Similarly, the fact that $m(D)$ does not belong to the space $\operatorname{span}\left\{g_{i j}, i+\right.$ $j>0\}$ is sufficient for $g\left(z_{1}, z_{2}\right)$ being different from zero for every $\left(z_{1}, z_{2}\right) \in$ $\mathbf{C} \times \mathbf{C}$, and then for $G\left(z_{1}, z_{2}\right)$ being zero-prime.

\section{Delayed inverses of 2D polynomial matrices}

In the previous sections we discussed several problems connected with the existence and the construction of polynomial inverses for an assigned transfer matrix $G\left(z_{1}, z_{2}\right)$. Here we confine ourselves to polynomial matrices, and we analyze the case in which $G\left(z_{1}, z_{2}\right)$ is full column rank over $\mathbf{R}\left(z_{1}, z_{2}\right)$ but not zero-prime.

Under this hypothesis, $G\left(z_{1}, z_{2}\right)$ admits rational, but not polynomial, inverses, and causality and stability issues, which have a trivial solution when a polynomial inverse exists, become now very relevant.

Both stability and causality of $G^{-1}\left(z_{1}, z_{2}\right)$ reduce to a set of conditions on the variety of the poles of $G^{-1}\left(z_{1}, z_{2}\right)$. Indeed, as far as stability is concerned, the denominators of the (irreducible) entries of $G^{-1}\left(z_{1}, z_{2}\right)$ must be devoid of zero on the unit polydisc, $\mathcal{P}_{1}:=\left\{\left(z_{1}, z_{2}\right):\left|z_{1}\right| \leq 1,\left|z_{2}\right| \leq 1\right\}$, whereas, when a causal inverse is needed, they must only be different from zero at the origin of $\mathbf{C} \times \mathbf{C}$, (i.e. $G(0,0)$ must be full column rank).

These properties will be analyzed in this section, and related to some conditions on the variety $\mathcal{V}(G)$ of the maximal order minors [13] of $G\left(z_{1}, z_{2}\right)$. 
When $G\left(z_{1}, z_{2}\right)$ does not admit causal inverses, it is a matter of great interest to state if there are at least delayed proper inverses, that is proper rational matrices $H\left(z_{1}, z_{2}\right)$ satisfying (1.2). Also the existence of such inverses will be connected with some geometrical properties of $\mathcal{V}(G)$.

Throughout this section $G\left(z_{1}, z_{2}\right)$ will be a $2 \mathrm{D}$ polynomial matrix $p \times m$, with $p>m$ and full (column) rank.

Proposition 6. Let $G\left(z_{1}, z_{2}\right)$ be a 2 D polynomial matrix and $\mathcal{V}(G)$ the variety of the maximal order minors of $G\left(z_{1}, z_{2}\right)$. Then:

a) every point $P \in \mathcal{V}(G)$ is a pole of all the left inverses of $G\left(z_{1}, z_{2}\right)$;

b) given a polynomial $p\left(z_{1}, z_{2}\right)$ having decomposition

$$
p\left(z_{1}, z_{2}\right)=p_{1}\left(z_{1}, z_{2}\right) \ldots p_{r}\left(z_{1}, z_{2}\right)
$$

into distinct irreducible factors, such that

$$
\begin{gathered}
\mathcal{V}(G) \subseteq \mathcal{V}(p) \\
\mathcal{V}(G) \nsubseteq \mathcal{V}\left(\prod_{i=1, i \neq j}^{r} p_{i}\right) \quad j=1, \ldots, r
\end{gathered}
$$

there is a left inverse of $G\left(z_{1}, z_{2}\right), G^{-1}\left(z_{1}, z_{2}\right)$, such that $\mathcal{V}(p)$ is the variety of the poles of $G^{-1}\left(z_{1}, z_{2}\right)$.

\section{PROOF}

a) Let

$$
G^{-1}\left(z_{1}, z_{2}\right)=\left[\frac{n_{i j}\left(z_{1}, z_{2}\right)}{d_{i j}\left(z_{1}, z_{2}\right)}\right]=\frac{\left[t_{i j}\left(z_{1}, z_{2}\right)\right]}{d\left(z_{1}, z_{2}\right)}
$$

be a left inverse of $G\left(z_{1}, z_{2}\right)$, with $n_{i j}\left(z_{1}, z_{2}\right) / d_{i j}\left(z_{1}, z_{2}\right)$ irreducible and $d\left(z_{1}, z_{2}\right)=1 . c . m .\left\{d_{i j}\left(z_{1}, z_{2}\right), i=1, \ldots, m, j=1, \ldots, p\right\}$, and let $D_{L}^{-1}\left(z_{1}, z_{2}\right) N_{L}\left(z_{1}, z_{2}\right)$ be a lcMFD of $G^{-1}\left(z_{1}, z_{2}\right)$.

Since $d\left(z_{1}, z_{2}\right)$ is the l.c.m.,

$$
\frac{\left(\operatorname{Adj} D_{L}\left(z_{1}, z_{2}\right)\right) N_{L}\left(z_{1}, z_{2}\right)}{\operatorname{det} D_{L}\left(z_{1}, z_{2}\right)}=\frac{\left[t_{i j}\right]}{d\left(z_{1}, z_{2}\right)}
$$

implies $d\left(z_{1}, z_{2}\right) \mid \operatorname{det} D_{L}\left(z_{1}, z_{2}\right)$.

Moreover, since $D_{L}\left(z_{1}, z_{2}\right)^{-1} N_{L}\left(z_{1}, z_{2}\right)$ is a lcMFD, from $D_{L}^{-1}\left(z_{1}, z_{2}\right) N_{L}\left(z_{1}, z_{2}\right)=$ $\left[d\left(z_{1}, z_{2}\right) I_{m}\right]^{-1}\left[t_{i j}\right]$, it follows that $\operatorname{det} D_{L}\left(z_{1}, z_{2}\right) \mid d^{m}\left(z_{1}, z_{2}\right)$. Consequently we have

$$
\mathcal{V}(d)=\mathcal{V}\left(\operatorname{det} D_{L}\right)
$$

As $D_{L}\left(z_{1}, z_{2}\right)=N_{L}\left(z_{1}, z_{2}\right) G\left(z_{1}, z_{2}\right)$, we obtain

$$
\mathcal{V}(G) \subseteq \mathcal{V}\left(\operatorname{det} D_{L}\right)=\mathcal{V}(d) .
$$


b) Denote by $G_{i}\left(z_{1}, z_{2}\right)=S_{i} G\left(z_{1}, z_{2}\right)$ the $i$-th $m \times m$ submatrix of $G\left(z_{1}, z_{2}\right)$, where $S_{i}$ is an appropriate selection matrix and $g_{i}\left(z_{1}, z_{2}\right)=\operatorname{det} G_{i}\left(z_{1}, z_{2}\right)$, $i=1, \ldots, N=\left(\begin{array}{c}p \\ m\end{array}\right)$.

By Hilbert's Nullstellensatz, there exist an integer $n$ and polynomials $\alpha_{i}\left(z_{1}, z_{2}\right)$, for $i=1, \ldots, N$, such that

$$
p^{n}\left(z_{1}, z_{2}\right)=\sum_{i=1}^{N} \alpha_{i}\left(z_{1}, z_{2}\right) g_{i}\left(z_{1}, z_{2}\right)
$$

From the identity $\operatorname{Adj}\left(S_{i} G\left(z_{1}, z_{2}\right)\right) S_{i} G\left(z_{1}, z_{2}\right)=g_{i}\left(z_{1}, z_{2}\right) I_{m}$, for $i=1, \ldots, N$, it follows that

$$
\left[\sum_{i=1}^{N} \alpha_{i}\left(z_{1}, z_{2}\right) \operatorname{Adj}\left(S_{i} G\left(z_{1}, z_{2}\right)\right) S_{i}\right] G\left(z_{1}, z_{2}\right)=p^{n}\left(z_{1}, z_{2}\right) I_{m}
$$

Then

$$
G^{-1}\left(z_{1}, z_{2}\right)=\frac{\sum_{i=1}^{N} \alpha_{i}\left(z_{1}, z_{2}\right) \operatorname{Adj}\left(S_{i} G\left(z_{1}, z_{2}\right)\right) S_{i}}{p^{n}\left(z_{1}, z_{2}\right)}
$$

is a left inverse of $G\left(z_{1}, z_{2}\right)$ that can be rewritten as $G^{-1}\left(z_{1}, z_{2}\right)=\frac{\left[t_{i j}\left(z_{1}, z_{2}\right)\right]}{d\left(z_{1}, z_{2}\right)}$, $\left\{t_{i j}\left(z_{1}, z_{2}\right), i=1, \ldots, m, j=1, \ldots, p ; d\left(z_{1}, z_{2}\right)\right\}$ being a set of coprime polynomials.

As $d\left(z_{1}, z_{2}\right) \mid p^{n}\left(z_{1}, z_{2}\right)$, it follows that

$$
d\left(z_{1}, z_{2}\right)=p_{1}^{n_{1}}\left(z_{1}, z_{2}\right) \ldots p_{r}^{n_{r}}\left(z_{1}, z_{2}\right) \quad 0 \leq n_{i} \leq n \quad i=1, \ldots, r
$$

and then

$$
\mathcal{V}(d) \subseteq \mathcal{V}(p)
$$

To prove that the varietes in (4.2) coincide, it will be enough to show that all the $n_{j}$ in (4.1) are strictly positive. By assuming that $n_{j}=0$, it follows that

$$
\mathcal{V}(G) \subseteq \mathcal{V}(d) \subseteq \mathcal{V}\left(\prod_{i=1, i \neq j}^{r} p_{i}\right),
$$

which contradicts the hypothesis

A transfer matrix $G\left(z_{1}, z_{2}\right)$ is stable if its entries, expressed as irreducible rational functions, have denominators devoid of zeroes in the closed unitary polydisc, $\mathcal{P}_{1}$. This condition implies, but is not equivalent to, BIBO stability, since $G\left(z_{1}, z_{2}\right)$ can have non essential singularities of the second kind on the distinguished boundary, $\mathcal{T}_{1}=\left\{\left(z_{1}, z_{2}\right) \in \mathbf{C} \times \mathbf{C}:\left|z_{1}\right|=\left|z_{2}\right|=1\right\}$, without loosing BIBO stability [14].

Nevertheless, the above conditions on the denominators of $G\left(z_{1}, z_{2}\right)$ are necessary and sufficient for $G\left(z_{1}, z_{2}\right)$ to be realizable by an internally stable state model (2.1). Since in this paper our interest is in the connections 
between transfer matrices and state models, it seems more natural to follow the definition introduced above. The condition for the existence of a stable inverse for $G\left(z_{1}, z_{2}\right)$ is then an immediate consequence of Proposition 6 .

Corollary : A 2D polynomial transfer matrix $G\left(z_{1}, z_{2}\right)$ admits a stable inverse if and only if $\mathcal{V}(G)$ has no intersections with the unit polydisc, $\mathcal{P}_{1}$.

\section{PROOF}

If $\mathcal{V}(G) \cap \mathcal{P}_{1} \neq \emptyset$, then any point of the intersection is be a pole of every left inverse of $G\left(z_{1}, z_{2}\right), G^{-1}\left(z_{1}, z_{2}\right)$. So every left inverse of $G\left(z_{1}, z_{2}\right)$ is unstable.

If $\mathcal{V}(G) \cap \mathcal{P}_{1}=\emptyset$ we can choose a polynomial $p\left(z_{1}, z_{2}\right)$ as in b) of Proposition 6 that satisfies the extra condition $\mathcal{V}(p) \cap \mathcal{P}_{1}=\emptyset$.

Any inverse of $G\left(z_{1}, z_{2}\right)$ having $\mathcal{V}(p)$ as the variety of the poles, is stable

When $G\left(z_{1}, z_{2}\right)$ does not admit proper inverses, the next proposition supplies necessary and sufficient conditions for the existence of delayed proper/polynomial inverses, based on the structure of the variety $\mathcal{V}(G)$.

Proposition 7. Let $G\left(z_{1}, z_{2}\right)$ be a 2 D polynomial matrix, and $\mathcal{V}(G)$ the variety of the maximal order minors of $G\left(z_{1}, z_{2}\right)$. Then

a) $G\left(z_{1}, z_{2}\right)$ has a delayed proper inverse , $H\left(z_{1}, z_{2}\right)$, if and only if every algebraic curve in $\mathcal{V}(G)$ passing through the origin of $\mathbf{C} \times \mathbf{C}$ is a subset of

$$
Z_{1} \cup Z_{2}=\left\{\left(z_{1}, z_{2}\right) \in \mathbf{C} \times \mathbf{C}: z_{1} z_{2}=0\right\} .
$$

b) In particular, $G\left(z_{1}, z_{2}\right)$ has a delayed polynomial inverse if and only if $\mathcal{V}(G)$ is a subset, possibly empty, of $Z_{1} \cup Z_{2}$.

\section{PROOF}

a) Assume that $G\left(z_{1}, z_{2}\right)$ admits a delayed proper inverse, i.e. a proper rational matrix $H\left(z_{1}, z_{2}\right)$ satisfying

$$
H\left(z_{1}, z_{2}\right) G\left(z_{1}, z_{2}\right)=z_{1}^{\nu_{1}} z_{2}^{\nu_{2}} I_{m}
$$

and let $D_{L}\left(z_{1}, z_{2}\right)^{-1} N_{L}\left(z_{1}, z_{2}\right)$ be a lcMFD of $H\left(z_{1}, z_{2}\right)$.

Rewriting (4.3) as

$$
N_{L}\left(z_{1}, z_{2}\right) G\left(z_{1}, z_{2}\right)=z_{1}^{\nu_{1}} z_{2}^{\nu_{2}} D_{L}\left(z_{1}, z_{2}\right)
$$

and taking the determinant on both sides of (4.4) gives

$$
\operatorname{det}\left[N_{L}\left(z_{1}, z_{2}\right) G\left(z_{1}, z_{2}\right)\right]=z_{1}^{m \nu_{1}} z_{2}^{m \nu_{2}} \operatorname{det} D_{L}\left(z_{1}, z_{2}\right)
$$


Since det $D_{L}(0,0) \neq 0$, the variety of the right hand side of (4.5) does not include algebraic curves passing through $(0,0)$ which are not subset of $Z_{1} \cup Z_{2}$.

This is also true for $\mathcal{V}\left(\operatorname{det}\left(N_{L} G\right)\right)$ and $\mathcal{V}(G)$.

To prove the converse assume that $\mathcal{V}(G)$ consists of distinct algebraic curves $\mathcal{V}\left(p_{1}\right), \ldots, \mathcal{V}\left(p_{h}\right)$ satisfying the hypothesis of the theorem and, possibly, a finite set of isolated points $M_{1}, \ldots, M_{k}$. Let $q_{i}\left(z_{1}, z_{2}\right)$ be irreducible polynomials that interpolate $M_{i}, i=1,2, \ldots, k$ and whose variety is either a coordinate axis or an algebraic curve that does not include $(0,0)$. Clearly

$$
p\left(z_{1}, z_{2}\right)=p_{1}\left(z_{1}, z_{2}\right) \ldots p_{h}\left(z_{1}, z_{2}\right) q_{1}\left(z_{1}, z_{2}\right) \ldots q_{k}\left(z_{1}, z_{2}\right)
$$

can be rewritten as

$$
p\left(z_{1}, z_{2}\right)=z_{1}^{n_{1}} z_{2}^{n_{2}} r\left(z_{1}, z_{2}\right)
$$

with $r(0,0) \neq 0$, and satisfies $\mathcal{V}(p) \supseteq \mathcal{V}(G)$.

Using the same notations as in the proof of Proposition 6, we have

$$
p^{n}\left(z_{1}, z_{2}\right)=\sum_{i=1}^{N} \alpha_{i}\left(z_{1}, z_{2}\right) \operatorname{Adj}\left[S_{i} G_{i}\left(z_{1}, z_{2}\right)\right] G\left(z_{1}, z_{2}\right)=z_{1}^{n n_{1}} z_{2}^{n n_{2}} r^{n}\left(z_{1}, z_{2}\right) I_{m} .
$$

Therefore

$$
r^{-n}\left(z_{1}, z_{2}\right)\left[\sum_{i=1}^{N} \alpha_{i}\left(z_{1}, z_{2}\right) \operatorname{Adj}\left[S_{i} G_{i}\left(z_{1}, z_{2}\right)\right] S_{i}\right]=H\left(z_{1}, z_{2}\right) I_{m}
$$

is a delayed proper inverse of $G\left(z_{1}, z_{2}\right)$.

b) If $H\left(z_{1}, z_{2}\right)$ in (4.3) is a polynomial matrix, $z_{1}^{-\nu_{1}} z_{2}^{-\nu_{2}} H\left(z_{1}, z_{2}\right)$ is a left inverse of $G\left(z_{1}, z_{2}\right)$ and its poles coincide with the elements of $Z_{1} \cup Z_{2}$. Therefore, by Proposition 6, $\mathcal{V}(G) \subset Z_{1} \cup Z_{2}$.

Conversely, if $\mathcal{V}(G) \subset Z_{1} \cup Z_{2}$, the proof of a) can be carried out with $p\left(z_{1}, z_{2}\right)=z_{1}^{n_{1}} z_{2}^{n_{2}}$ and, therefore, $H\left(z_{1}, z_{2}\right)$ in (4.6) is a polynomial matrix

\section{Conclusions}

In this paper we have considered the question of existence, properties and construction of an inverse for a $2 \mathrm{D}$ system. Intuitively speaking, such an 
inverse is a proper $2 \mathrm{D}$ system which, when cascaded with the original one, reproduces as its output the input (possibly delayed) to the original system. Natural implications of invertibility theory are easily found in 2D linear decoupling and noninteracting control [1]. Further questions, regarding the invertibility of $2 \mathrm{D}$ dynamical models, have recently arised [2] in connection with the design of $2 \mathrm{D}$ convolutional encoders and decoders.

The approach developed in this paper is based both on the transfer function analysis and the state space realization procedures. Tha main results can be summarized as follows. First, necessary and sufficient conditions have been proved guaranteeing that a polynomial transfer matrix $G\left(z_{1}, z_{2}\right)$ admits a (possibly delayed) polynomial inverse. This result, whose relevance for applications to convolutional decoding is quite obvious, has been further extended, thus providing conditions for the existence of proper and stable rational inverses.

Second, the possibility of obtaining a finite memory inverse system of a given realization of $G\left(z_{1}, z_{2}\right)$ has been investigated. Solving this problem is quite appealing, since it allows to synthesize at the same time a good encoder and a reliable decoder by designing essentially a unique 2D system.

\section{References}

1. E.Fornasini, G.Marchesini - Noninteracting control of $2 D$ systems IEEE Trans. on Aut.Contr., vol. AC 36, pp.90-95, 1991

2. E.Fornasini, M.E.Valcher - Some aspects of the algebraic structure of 2D convolutional codes - Proc. Eurocode 92, CISM, Udine (Italy) 1992

3. E.Fornasini, G.Marchesini - Doubly indexed dynamical systems - Math.Sys. Theory, vol.12, pp.59-72, 1978

4. R.P.Roesser - A discrete state space model for linear image processing - IEEE Trans. on Aut.Contr., vol.AC 20, pp.1-10, 1975

5. J.L.Massey - M.K.Sain - Inverses of linear sequential circuits - IEEE Trans.on Comp., vol.C 17, pp.330-337, 1968

6. M.K.Sain - J.L.Massey - Invertibility of linear time invariant dynamical systems - IEEE Trans.on Aut.Contr., vol.AC 14, pp141-149, 1969

7. M.Bisiacco - State and output feedback stabilizability of $2 D$ systems IEEE Trans. Circ. and Sys., vol.CAS 32, pp.1246-1249, 1985 
8. E.Fornasini - S.Zampieri - A note on the state space realization of $2 D$ FIR transfer functions - Sys. and Contr.Letters, vol.16, pp.17-22, 1990

9. M.Morf - B.C.Levy - S.Y.Kung - New results in 2D systems theory, Part I and II - Proc. of IEEE, vol.65, no.6, pp.861-872, 1977

10. D.C.Youla - G.Gnavi - Notes on n-dimensional system theory - IEEE Trans.Circ. and Sys., vol.CAS 26, pp.105-111, 1979

11. D.C.Youla - P.F.Pickel - The Quillen-Suslin theorem - IEEE Trans.Circ. and Sys., vol.CAS 31, pp.513-518, 1984

12. E.Fornasini - M.E.Valcher - On the structure of finite memory and separable 2D systems - Proc. 2nd IFAC Workshop on System Structure and Control, Prague, Sept.1992

13. M.Bisiacco - E.Fornasini - G.Marchesini - Dynamic Regulation of $2 D$ systems: a state approach - Lin. Algebra and Appl. 122-124, pp.195218,1989

14. D.Goodman - Some stability properties of two-dimensional linear shiftinvariant digital filters - IEEE Trans. Circ. and Sys., vol.CAS 24, pp.201-208, 1977 